\title{
100 Things You Always Wanted to Know about Semantics \& Pragmatics But Were Afraid to Ask*
}

\author{
Emily M. Bender \\ University of Washington \\ Department of Linguistics \\ Box 3542425 \\ Seattle WA 98195-2425 USA \\ ebender@uw. edu
}

\section{Tutorial overview}

Meaning is a fundamental concept in Natural Language Processing (NLP), given its aim to build systems that mean what they say to you, and understand what you say to them. In order for NLP to scale beyond partial, task-specific solutions, it must be informed by what is known about how humans use language to express and understand communicative intents. The purpose of this tutorial is to present a selection of useful information about semantics and pragmatics, as understood in linguistics, in a way that's accessible to and useful for NLP practitioners with minimal (or even no) prior training in linguistics.

The tutorial will look at both aspects of meaning tied to the linguistic signal (sentence meaning), including how it is tied to syntactic structure, and aspects of meaning in situated language use (speaker meaning). For the most part, the points will be illustrated with English examples, but to the extent possible I will bring in a typological perspective to foster an understanding of to what extent phenomena are crosslinguistically variable and to highlight semantic phenomena that are not present in English.

The tutorial will briefly cover the following six topics:

1. Introduction: What is meaning? What is the difference between speaker meaning and sentence meaning? How do they relate to the tasks of interest to participants?

2. Lexical semantics: What do words mean? What kind of formally precise devices allow for compact representations and tractable inference with word meanings? How are those meanings related to each other? How do those meanings change over time?
3. Semantics of phrases: How do we build the meaning of the phrase from the meaning of the parts? How should one tackle (possibly partially) non-compositional elements like multi-word expressions?

4. Meaning beyond the sentence: How do sentences in discourse relate to each other? How do we connect referring expressions with the same referents?

5. Presupposition and implicature: What are presuppositions and implicatures? What linguistic expressions introduce presuppositions and how do they interact in larger structures? How do we calculate implicatures?

6. Resources: What linguistic resources have been built to assist with semantic processing?

\section{Instructor}

Emily M. Bender is a Professor in the Department of Linguistics and Adjunct Professor in the Paul G. Allen School of Computer Science \& Engineering at the University of Washington. She is also the past chair (2016-2017) of NAACL. Her research interests lie in multilingual grammar engineering, computational semantics, and the incorporation of linguistic knowledge in natural language processing. She is the PI of the Grammar Matrix grammar customization system, which is developed in the context of the DELPH-IN Consortium (Deep Linguistic Processing with HPSG Initiative). More generally, she is interested in the intersection of linguistics and computational linguistics, from both directions: bringing computational methodologies to linguistic science and linguistic science to natural language processing.

*.. for fear of being told 1000 more 